\title{
Composition en polyphénols totaux et en tanins des feuilles de neuf variétés de Cajanus cajan (L.) Millsp. au cours du premier cycle de croissance et en fonction du mode d'exploitation
}

\author{
Faustin Parfait KOUTOUAN ${ }^{1 *}$, Yapo Magloire YAPI $^{2}$, Eboua Narcisse WANDAN ${ }^{3}$, \\ Nguessan Clément BODJI ${ }^{2}$ et Kessé Phillipe N'DA ${ }^{4}$
}
${ }^{1}$ Institut National Polytechnique-Félix Houphouët Boigny de Yamoussoukro. Laboratoire Sciences Société \& Environnement, UMRI Sciences Agronomiques et Génie Rurale, BP 1093, Yamoussoukro.
${ }^{2}$ Institut National Polytechnique-Félix Houphouët Boigny de Yamoussoukro, Département Agriculture et Ressources Animales, Laboratoire de Zootechnie et Productions Animales, BP 1313, Yamoussoukro. ${ }^{3}$ Institut National Polytechnique-Félix Houphouët Boigny de Yamoussoukro, Laboratoire Sciences Société \& Environnement, UMRI Sciences Agronomiques et Génie Rurale, BP 1093 Yamoussoukro.
${ }^{4}$ Institut National Polytechnique-Félix Houphouët Boigny de Yamoussoukro, Département de Recherche et de Formation Génie Chimique et Agro-alimentaire, Laboratoire de Chimie, de l'Eau et des Substances Naturelles, BP 1093, Yamoussoukro.
*Auteur correspondant, E-mail: koutouanparfait@gmail.com

\section{RESUME}

Le pois d'angole est une légumineuse fourragère et vivrière dotée de propriétés anthelminthiques dues à ses métabolites secondaires. En vue de caractériser les teneurs en composés polyphénoliques de neuf variétés au cours des stades végétatifs, une étude a été menée. Les variétés ont été semées à raison de huit lignes par variété. Quatre lignes ont été fauchées trois mois après semis et quatre autres sont restées en l'état. Les polyphénols totaux (PT), les tanins totaux (TT) et les tanins condensés (TC) ont été déterminés dans les feuilles des plants fauchés et des plants non fauchés au cours du cycle végétatif. Les PT et les TT ont été déterminés par la méthode de Folin Ciocalteu et les TC, par la méthode HCl-butanol. Les teneurs en PT, TT et TCs, tout

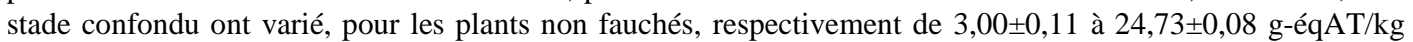
MS, $0,12 \pm 0,08$ à $11,42 \pm 0,24$ g-éqAT/kg MS et $0,01 \pm 0,00$ à $1,61 \pm 0,06$ g-éqL $/ \mathrm{kg} \mathrm{MS}$. Ces teneurs étaient

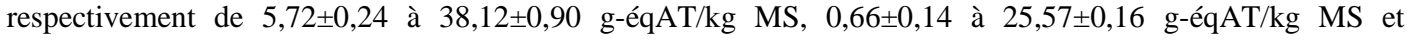
$0,04 \pm 0,00$ à $1,80 \pm 0,08 \mathrm{~g}$-éqL/kg MS pour les plants fauchés. La teneur en ces composés évolue au cours du cycle et est influencée par la fauche.

(C) 2019 International Formulae Group. All rights reserved

Mots clés: Pois d'Angole, métabolites secondaires, teneur, cycle végétatif.

\section{Concentration of polyphenols and tannins in the leaves of nine varieties of Cajanus cajan (L.) Millsp. during the growth cycle and according to the mode of exploitation}

\begin{abstract}
Pigeon pea is a shrub fodder legume with anthelmintic properties coming from its secondary metabolites content. This study aimed at characterizing the levels of polyphenols and tannins in nine varieties of Cajanus cajan during their cycle of development. The varieties of Cajanus cajan were sown in eight rows
\end{abstract}


per variety. Four lines were mowed at 3 months of age at the height of $100 \mathrm{~cm}$ from the ground. Folin Ciocalteu method was used to measure Total polyphenols (TPs), and total tannins (TT) in the leaves of mowed and unmown plants during their development stages. Total condensed tannins (TCs) were also measured by the HCl-butanol method. Levels of PTs found in unmown plants ranged from $3.00 \pm 0.11$ to $24.73 \pm 0.08$ eq-gTA $/ \mathrm{kg}$ DM while the levels in mown plants ranged from $5.72 \pm 0.24$ to $38.12 \pm 0.90$ eq-gTA $/ \mathrm{kg}$ DM. The concentration of TTs ranged from $0.12 \pm 0.08$ to $11.42 \pm 0.24$ eq-gTA $/ \mathrm{kg}$ DM in unmown plants, and from $0.66 \pm 0.14$ eqgTA $/ \mathrm{kg}$ DM to $25.57 \pm 0.16$ eq-gTA $/ \mathrm{kg} \mathrm{DM}$ in mown ones. The levels of CTs in unmown plants ranged from $0.01 \pm 0.00$ to $1.61 \pm 0.06 \mathrm{eq}-\mathrm{gL} / \mathrm{kg} \mathrm{DM}$ while they were found to ranges from $0.04 \pm 0.00 \mathrm{eq}-\mathrm{gL} / \mathrm{kg}$ to $1.80 \pm 0.08$ eq-gL/kg in mown plants. In general, the levels of polyphenols and tannins in $C$. cajan increased during the growing cycle, and were influenced by both variety and exploitation mode.

(C) 2019 International Formulae Group. All rights reserved

Keywords: Pigeon pea, secondary compounds, levels, vegetative cycle.

\section{INTRODUCTION}

Cajanus cajan $\mathrm{L}$. est une légumineuse arbustive fourragère et vivrière qui s'intègre bien en Afrique dans les systèmes de production culture-bétail (Onana et al., 2007 ; Pal et al., 2011). Elle produit une grande quantité de biomasse fourragère de haute qualité (Fondevila et al., 2002; Shenkute et al., 2013) et est également utilisée dans le traitement de certaines pathologies humaines et animales (Pal et al., 2011; Ogni et al., 2014; N'draman-donou et al., 2015). Sur le plan parasitaire, l'action de Cajanus cajan contre les nématodes gastro-intestinaux (Pal et al., 2011; Kaboré et al., 2016) est à ce jour documentée. La présence dans cette plante de flavonoïdes et de polyphénols (Harris et al., 2014; Vidhya et Ahmed, 2017), particulièrement les tanins condensés (Fondevila et al., 2002) serait la source de ces propriétés anthelminthiques (Pal et al., 2011; Kaboré et al., 2016). A ce jour, l'effet des tanins condensés sur les nématodes gastrointestinaux est prouvé (Hoste et al., 2006). Cependant, malgré les qualités fourragère et anthelminthique de Cajanus cajan, la littérature reste très peu pourvue sur cette espèce en ce qui concerne ses propriétés nutraceutiques, c'est-à-dire sa possibilité à combiner à la fois des propriétés nutritionnelle et anthelminthique (Hoste et al., 2015). Dans les travaux de Kaboré et al. (2016) qui traitent de ce sujet, l'activité anthelminthique n'a pas été significative, la variété et le stade phénologique n'ont pas non plus été précisés, alors que les polyphénols et les tanins condensés, supposés responsables de l'activité anthelminthique varient avec la variété et le stade phénologique (Azuhnwi et al., 2011; Girard et al., 2013). Même dans les tests in vitro où l'effet anthelminthique est mis en évidence ( $\mathrm{Pal}$ et al., 2011; Kaboré et al., 2016), les variétés et les stades phénologiques n'ont pas été non plus précisés, alors que Cajanus cajan regorge une multitude de variétés, aussi bien traditionnelles qu'améliorées ( Yadav et al., 2012).

L'étude des propriétés nutraceutiques de cette espèce passe donc par la connaissance de l'évolution, en fonction des variétés et du stade phénologique, de la teneur en polyphénols et en tanins, lesquels suivant leurs teneurs dans la plante ont des effets bénéfiques ou néfastes sur la valeur alimentaire $\mathrm{du}$ fourrage et sur les performances des ruminants (Seresinhe et Pathirana, 2003). L'étude de la teneur en polyphénols et en tanins doit prendre, également en compte le mode d'exploitation, car l'exploitation de Cajanus cajan en tant que culture vivrière et fourragère, nécessite de développer une stratégie d'exploitation. Certains agro-éleveurs fauchent deux fois de suite les plants de cette espèce à des fins fourragères avant de récolter les graines pour l'autoconsommation, puis la ré-exploitent à nouveau dans un but fourrager (Banfoh, 2004). 
Cette étude est menée afin d'étudier les teneurs en polyphénols totaux et en tanins de neuf variétés de Cajanus cajan d'origine diverses et cultivées sur le même site au cours de la première année de culture en rapport avec le mode d'exploitation.

\section{MATERIEL ET METHODES Zone d'étude}

L'étude a été conduite dans la région centre de la Côte d'Ivoire, précisément dans le District Autonome de Yamoussoukro. L'expérience proprement dite s'est déroulée à la Station Semencière de Yamoussoukro (6 ${ }^{\circ} 54^{\prime} 35,66^{\prime \prime} \mathrm{N}$ de latitude ; $5^{\circ} 30^{\prime} 57,21^{\prime \prime} \mathrm{O}$ de longitude), sur un sol de type ferrasol avec un pH de 6,5, composé majoritairement de sable $(77,25 \%)$ et de limon $(15,75 \%)$ avec $1,7 \%$ de carbone organique (Koutouan et al., 2017). La pluviosité annuelle moyenne enregistrée de 2006 à 2017 sur cette station est de 1 151,3 $\mathrm{mm}$.

\section{Matériel végétal}

Cinq variétés améliorées, une variété traditionnelle et trois cultivars traditionnels de Cajanus cajan de diverses origines ont été utilisés pour l'étude. Leurs caractéristiques sont consignées dans le Tableau 1.

\section{Dispositif expérimental et collecte des échantillons}

Les semis des variétés et cultivars ont été réalisés à la grande saison pluvieuse, notamment en mai 2017. Les graines ont été mises en poquets à raison de trois graines par poquet. Deux sarclages ont été effectués les $32^{\mathrm{e}}$ et $68^{\mathrm{e}}$ jours après le semis (JAS). Le démariage a été réalisé au $45^{\mathrm{e}} \mathrm{JAS}$ de sorte à avoir un pied par poquet. Aucun fertilisant ni pesticide n'a été utilisé.

Chaque variété a été semée sur des lignes à raison de huit lignes par variété. Sur chaque ligne, 15 poquets ont été ensemencés. Les écartements de $1 \mathrm{~m}$ en tous sens ont été utilisés. Sur les huit lignes par variété, quatre ont été fauchées trois mois après semis à une hauteur de $100 \mathrm{~cm}$ du sol tel que recommandée par Bodji (1997) et quatre autres sont restées en l'état (Figure 1). Pour l'identification des plants sur lesquels les feuilles ont été prélevées, la parcelle a été virtuellement divisée en trois parties selon le sens de la pente: haut de pente, milieu de pente et bas de pente. Par variété, deux plants ont été choisis au hasard sur les lignes centrales dans chaque zone ainsi délimitée, aussi bien au niveau des lignes fauchées que des lignes non fauchées. Ce qui fait un total de six plants pour la bande fauchée et six autres pour la bande non fauchée, soit un total 12 plants par variété.

A chaque stade, les feuilles ont été prélevées sur toute la plante. Pour la phase végétative, les prélèvements ont été effectués pour toutes les variétés à 90 JAS. Pour les autres phases, le prélèvement s'est fait selon le cycle de la variété ou du cultivar. Le stade de floraison a été considéré comme atteint, lorsqu'il y avait présence des premières fleurs et absence des gousses. De même, le stade fructification a été atteint lorsqu'on a observé les premières gousses immatures. Enfin le stade maturation a été atteint lorsque les premières gousses sont arrivées à maturité.

Les feuilles collectées ont été transportées dans des sacs laissant passer l'air, aussitôt que possible et séchées sous abri pendant au moins 21 jours dans une salle aérée. Elles ont par la suite été broyées à l'aide d'un Blender de marque PHILIPS, puis les poudres obtenues ont été placées dans des sachets noirs et conservées dans une pièce sèche et sombre à température ambiante jusqu'à la période d'analyse.

\section{Extraction}

A $0,2 \mathrm{~g}$ de poudre végétale, ont été ajoutés $10 \mathrm{ml}$ d'une solution eau-acétone (30:70 v/v). Le mélange obtenu a été mis en suspension dans un bain d'eau à ultrasons pendant $30 \mathrm{~min}$ (deux fois $15 \mathrm{mn}$ ) à la température ambiante. Le contenu a été ensuite soumis à une centrifugation pendant 10 minutes. Le surnageant a été recueilli et gardé sous la glace jusqu'à la fin des analyses. 
Pour le calcul du rendement d'extraction, le même protocole jusqu'au traitement par l'ultrason a été repris sur un autre échantillon de poudre végétale du stade maturation. L'extrait liquide obtenu a été filtré à l'aide de coton à laine. Le filtrat liquide obtenu a été mis à l'étuve à $50{ }^{\circ} \mathrm{C}$ jusqu'à la dessiccation totale. L'extrait sec obtenu a permis de calculer le rendement d'extraction

\section{Calcul du rendement d'extraction}

Le rendement d'extraction (R) a été obtenu grâce à la formule suivante :

$\mathrm{R}(\%)$

$$
=\frac{\text { Extrait sec obtenu } \times 100}{\text { quantité de poudre végétale utilisée }}
$$

\section{Détermination des teneurs en composés phénoliques}

Les polyphénols totaux (PT), les tanins totaux (TT) et les tanins condensés (TC) ont été déterminés sur les plants fauchés et sur les plants non fauchés aux stades végétatifs, floraison, fructification et maturation.

Les PT ont été déterminés par la méthode de Folin Ciocalteu décrite par Makkar (2000). A $25 \mu \mathrm{L}$ de l'extrait liquide est ajoutée de l'eau distillée jusqu'à l'obtention de $0,5 \mathrm{ml}$. Puis sont ajoutés 0,25 $\mathrm{ml}$ de solution de Folin-Ciocalteu $(1 \mathrm{~N})$ et $1,25 \mathrm{ml}$ de solution de carbonate de sodium (20\%). Le mélange a été vortexé et l'absorbance a été lue à $725 \mathrm{~nm}$ après $40 \mathrm{~min}$ d'incubation à la température ambiante et à l'obscurité. La teneur en PT déterminée à l'aide d'une droite d'étalonnage réalisée avec de l'acide tannique de Merck a été exprimée en gramme équivalent acide tannique par kilogramme de matière sèche (g-éqAT/kg MS).

Les tanins totaux (TT) ont été estimés indirectement après avoir été absorbés par la polyvinylpyrrolidine insoluble (PVP). Une solution comprenant $100 \mathrm{mg}$ de PVP, $1,0 \mathrm{ml}$ d'eau distillée et $1,0 \mathrm{ml}$ de l'extrait a été constituée. L'ensemble a été vortexé puis gardé à $4{ }^{\circ} \mathrm{C}$ pendant 15 min. Il a été par la suite centrifugé pendant $10 \mathrm{~min}$ et le surnageant a été ensuite recueilli. La teneur en polyphénols totaux (ne contenant pas les tanins) a été déterminée sur $50 \mu \mathrm{L}$ de ce surnageant. La concentration des TT a été déterminée comme la différence en PT avant et après le traitement avec la PVP insoluble.

Les tannins condensés ont été déterminés par la méthode de HCl-Butanol décrite par Makkar (2000). Une quantité de $0,50 \mathrm{ml}$ de l'extrait dilué avec70\% d'acétone a été prélevé puis 3,0 $\mathrm{ml}$ du réactif $\mathrm{HCl}$-butanol et $0,1 \mathrm{ml}$ du réactif ferrique y ont été ajoutés et vortexé. Le tout a été mis dans un bain d'eau bouillante pendant $60 \mathrm{~min}$ puis refroidi. Les absorbances ont été lues à $550 \mathrm{~nm}$. La teneur en tanins condensés, exprimée en g-éq leucocyanidine $/ \mathrm{kg}$ MS, est donnée par la formule suivante :

$$
T C=\frac{A_{550 \mathrm{~nm}} \times 78,26 \times 0,5}{\% \text { matière sèche }}
$$

\section{Analyses statistiques}

L'analyse de variance (ANOVA) à un critère de classification au seuil de $1 \%$ a été utilisée pour la comparaison des moyennes des différents composés phénoliques au cours du cycle de développement de la variété et pour la comparaison entre variété pour chaque stade de développement. Lorsqu'il y avait une différence significative, le test de comparaison multiple de Newman-Keuls au seuil de $1 \%$ a été appliqué.

Ensuite, l'ANOVA à deux critères de classification au seuil de $1 \%$ a été utilisée pour les interactions entre les variétés et le mode d'exploitation pour les différents composés phénoliques à chaque stade phénologique. Lorsque l'interaction était significative, des tests $t$ de Student ont été effectués pour comparer deux à deux les moyennes afin de localiser le lieu de l'interaction. Toutes les analyses ont été faites avec le logiciel XLSTAT version 2014. 
Tableau 1: Caractéristiques des variétés et cultivars utilisés.

\begin{tabular}{|c|c|c|c|}
\hline Variétés/Cultivars & $\begin{array}{l}\text { Couleur de } \\
\text { la semence }\end{array}$ & $\begin{array}{c}\text { Lieu d'achat de la } \\
\text { semence }\end{array}$ & Nature de la variétés/cultivars \\
\hline Cultivar local blanc & Blanche & $\begin{array}{l}\text { Marché local de } \\
\text { Bondoukou (Côte } \\
\text { d'Ivoire) }\end{array}$ & $\begin{array}{l}\text { Cultivar local de la Côte } \\
\text { d'Ivoire }\end{array}$ \\
\hline Cultivar local rouge & Rouge & $\begin{array}{l}\text { Marché local de } \\
\text { Bondoukou (Côte } \\
\text { d'Ivoire) }\end{array}$ & $\begin{array}{l}\text { Cultivar local de la Côte } \\
\text { d'Ivoire }\end{array}$ \\
\hline Cultivar local noir & Noire & $\begin{array}{l}\text { Marché local de } \\
\text { Bondoukou (Côte } \\
\text { d'Ivoire) }\end{array}$ & $\begin{array}{l}\text { Cultivar local de la Côte } \\
\text { d'Ivoire }\end{array}$ \\
\hline $\begin{array}{l}\text { ICPL } 87 \text { 119- } \\
\text { ASHA }\end{array}$ & Rouge & $\begin{array}{l}* * \text { INERA, Burkina } \\
\text { Faso }\end{array}$ & Variété améliorée \\
\hline ICP 8863-MARUTI & Rouge & $\begin{array}{l}\text { INERA, Burkina } \\
\text { Faso }\end{array}$ & Variété améliorée \\
\hline $\begin{array}{l}\text { ICP 7035- } \\
\text { KAMICA }\end{array}$ & Rouge & $\begin{array}{l}\text { INERA, Burkina } \\
\text { Faso }\end{array}$ & Variété améliorée \\
\hline FKB-ROUGE & Rouge & $\begin{array}{l}\text { INERA, Burkina } \\
\text { Faso }\end{array}$ & Variété locale du Burkina Faso \\
\hline $\begin{array}{l}\text { ICPL } 87119 \\
\text { GUIMU } 3 \text { (Asha) }\end{array}$ & Rouge & $\begin{array}{l}\text { INERA, Burkina } \\
\text { Faso }\end{array}$ & Variété améliorée \\
\hline $\begin{array}{l}\text { ICPL } 332 \\
\text { ABAAYA }\end{array}$ & Rouge & $\begin{array}{l}\text { INERA, Burkina } \\
\text { Faso }\end{array}$ & Variété améliorée \\
\hline
\end{tabular}

**INERA= Institut National de l'Environnement et de la Recherche Agronomique.

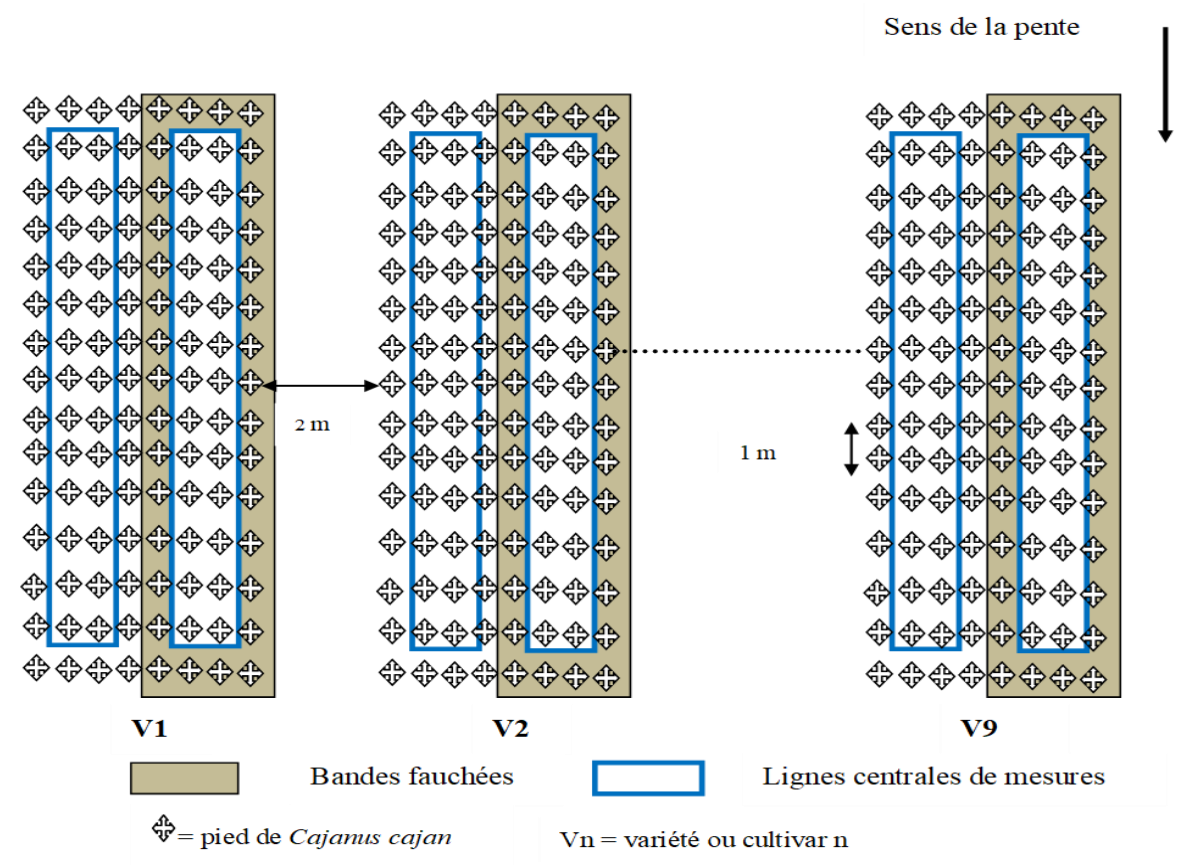

Figure 1: Dispositif expérimental. 


\section{RESULTATS}

\section{Rendements d'extraction}

Les rendements d'extraction sont consignés dans le Tableau 2. Ils varient de 20,23 à $24,86 \%$.

\section{Evolution des teneurs en composés phénoliques des variétés de $C$. cajan au cours du premier cycle de développement de Cajanus cajan}

La teneur en polyphénols totaux des différentes variétés a varié de $3,00 \pm 0,11$ à $24,73 \pm 0,08 \mathrm{~g}$-éqAT/kg MS pour les plants non fauchés (Figure 2). Pour chaque stade phénologique, une différence significative $(\mathrm{P}<0,01)$ existe entre les variétés et les cultivars de $C$. cajan de l'étude. Le cultivar local blanc a obtenu les concentrations en polyphénols totaux les plus élevées aux stades végétatif et fructification (respectivement $5,59 \pm 0,09$ et $23,17 \pm 1,12$ g-éqAT/kg MS), tandis qu'aux stades floraison et maturation, c'est le cultivar local noir qui a enregistré les teneurs les plus importantes (respectivement $17,83 \pm 0,56$ et $24,73 \mathrm{~g}$-éqAT/kg MS). Toutes les variétés et cultivars ont connu une augmentation de leur teneur en polyphénols totaux du stade végétatif au stade maturation. Cependant, selon les variétés et cultivars, la teneur maximale est obtenue soit en fructification (cas des variétés ICP 8863MARUTI, ICP 7035-KAMICA et ICPL 332 ABAAYA), ou soit en maturation (cas des cultivars locaux et des variétés ICPL 87 119ASHA, FKB-ROUGE et ICPL 87119 GUIMU 3 (Asha)). Toutefois, pour le cultivar local blanc, la différence constatée entre le stade fructification et le stade maturation n'est pas significative $(\mathrm{P}>0,01)$.

Pour les tanins totaux, leurs teneurs sont situées entre $0,12 \pm 0,08$ et $11,42 \pm 0,24 \mathrm{~g}$ éqAT/kg MS (Figure 3). Une différence significative $(\mathrm{P}<0,01)$ existe entre les variétés et les cultivars aux différents stades phénologiques. Aux stades végétatif et floraison, le cultivar local rouge a enregistré les teneurs en tanins totaux les plus élevées avec respectivement $2,96 \pm 0,10$ et 7,26 $\pm 0,62$ g-éqAT/kg MS. Le cultivar local blanc a obtenu les teneurs les plus élevées aux stades fructification et maturation avec respectivement $9,87 \pm 0,54$ et $11,42 \pm 0,24 \mathrm{~g}$ éqAT/kg MS. Toutes les variétés et cultivars ont connu une augmentation de leur teneur en tanins totaux du stade végétatif au stade maturation. Cependant, selon les variétés, la teneur maximale est obtenue soit en fructification (cas des variétés ICP 8863MARUTI, ICP 7035-KAMICA et ICPL 332 ABAAYA), soit en maturation (cas des cultivars locaux et des variétés ICPL 87 119ASHA, FKB-ROUGE et ICPL 87119 GUIMU 3 (Asha)).

Enfin, pour les tanins condensés, les teneurs des différentes variétés et cultivars ont oscillé de $0,01 \pm 0,00$ à $1,61 \pm 0,06 \mathrm{~g}$-éqL $/ \mathrm{kg}$ MS (Figure 4). Une différence significative $(\mathrm{P}<0,01)$ existe également entre les variétés et les cultivars aux différents stades phénologiques. Au stade végétatif, la variété FKB-ROUGE a enregistré la teneur en tanins condensés la plus importante $(1,05 \pm 0,02 \mathrm{~g}$ éqL/kg MS), tandis qu'au stade floraison, c'est le cultivar local noir qui a connu la plus forte concentration avec 1,34 $\pm 0,04 \mathrm{~g}$-éqL/kg MS. Le cultivar local blanc a obtenu les teneurs les plus élevées aux stades fructification et maturation avec respectivement $1,43 \pm 0,05$ et $1,61 \pm 0,06 \mathrm{~g}$ éqL/kg MS. Toutes les variétés et cultivars ont également connu une augmentation de leurs concentrations en tanins condensés du stade végétatif au stade maturation. Cependant, selon les variétés, la teneur maximale est obtenue soit en floraison (cas du cultivar local rouge), soit en fructification (cas des variétés ICP 7035-KAMICA, FKB-ROUGE, ICPL 87 119 GUIMU 3 (Asha) et ICPL 332 ABAAYA), ou soit encore en maturation (cas du cultivar local blanc, du cultivar local noir et des variétés ICPL 87 119-ASHA et ICP 8863-MARUTI).

\section{Interaction mode d'exploitation et variétés sur la teneur en polyphénols et en tanins}

La teneur en polyphénols totaux des plants fauchés des variétés et cultivars a varié de $5,72 \pm 0,24$ à $38,12 \pm 0,90$ g-éqAT/kg MS (Figure 5). Au stade floraison, les plants 
fauchés ont enregistré des teneurs en polyphénols plus élevées que les plants non fauchés chez toutes les variétés et cultivars à l'exception de la variété ICP 8863-MARUTI où aucune différence significative $(\mathrm{P}>0,01)$ n'est observée entre les plants fauchés et les plants non fauchés. Au stade fructification, pour certaines variétés et cultivars (cultivar local rouge, cultivar local noir et la variété ICP 7035-KAMICA), les plants fauchés ont présenté des teneurs plus importantes $(\mathrm{P}<0,01)$ que les plants non fauchés. Pour d'autres variétés (ICP 8863-MARUTI, FKBROUGE et ICPL 87119 Asha), aucune différence significative $(\mathrm{P}>0,01) \quad$ n'est constatée entre les plants fauchés et les non fauchés. Enfin, pour le cultivar local blanc et les variétés ICPL 87119 GUIMU 3 (Asha) et ICPL 332 ABAAYA, les plants fauchés ont produit moins de polyphénols que les plants non fauchés. Enfin, au stade maturation, pour les cultivars locaux rouge et blanc ainsi que la variété ICP 8863-MARUTI, les plants fauchés ont produit plus de polyphénols que les plants non fauchés ; ce qui est le contraire pour les variétés FKB-ROUGE, ICPL 87119 Asha et ICPL 87119 GUIMU 3 (Asha). Au niveau du cultivar local noir et des variétés ICP 7035KAMICA et ICPL 332 ABAAYA, aucune différence significative $(\mathrm{P}>0,01)$ n'est constatée entre les plants fauchés et les plants non fauchés.

La teneur en tanins totaux des plants fauchés est comprise entre $0,66 \pm 0,14$ et 25,57 $\pm 0,16$ g-éqAT/kg MS (Figure 6). Au stade floraison, à l'exception des variétés ICPL 332 ABAAYA, ICPL 87119 GUIMU 3 (Asha) et ICP 8863-MARUTI pour lesquelles aucune différence significative $(\mathrm{P}>0,01)$ n'est observée entre les plants fauchés et les plants non fauchés, les différentes variétés et cultivars ont enregistré une production plus élevée en tanins totaux chez les plants fauchés. Au stade fructification, pour les cultivars locaux rouge et noir ainsi que la variété FKB-ROUGE, les plants fauchés ont produit plus de tanins totaux que les plants non fauchés. Pour les variétés ICP 7035-
KAMICA et ICPL 87119 Asha, aucune différence significative $(\mathrm{P}>0,01) \quad$ n'est constatée entre les plants fauchés et les plants non fauchés. Enfin, pour le cultivar local blanc et les variétés ICP 8863-MARUTI, ICPL 87119 GUIMU 3 (Asha) et ICPL 332 ABAAYA, les plants fauchés ont enregistré des teneurs en tanins totaux inférieures à celles des plants non fauchés. Enfin, au stade maturation, seul le cultivar local noir a enregistré une teneur plus élevée chez les plants fauchés comparativement aux plants non fauchés. Au niveau des autres variétés et cultivars, soit aucune différence significative ( $P>0,01)$ n'est constatée pour le cultivar local rouge et les variétés ICP 8863-MARUTI, ICP 7035-KAMICA et ICPL 332 ABAAYA. Par contre, pour le cultivar local blanc et les variétés FKB-ROUGE, ICPL 87119 Asha et ICPL 87119 GUIMU 3 (Asha), les plants fauchés ont produit moins de tanins totaux.

Pour les tanins condensés, les valeurs des plants fauchés ont varié de $0,04 \pm 0,00$ à $1,80 \pm 0,08$ g-éqL/kg MS (Figure 7). Au stade floraison, les plants fauchés des cultivars locaux rouge et noir ainsi que des variétés FKB-ROUGE et ICPL 87119 Asha ont produit plus de tanins condensés comparativement aux plants non fauchés. Ce qui est le contraire chez les variétés ICPL 87 119 GUIMU 3 (Asha) et ICPL 332 ABAAYA, ICP 7035-KAMICA et ICP 8863MARUTI. Pour le cultivar local blanc, aucune différence significative $(\mathrm{P}>0,01) \quad$ n'est constatée entre les plants fauchés et non fauchés. Au stade fructification, les cultivars locaux rouge et noir ont présenté des teneurs plus importantes en tanins condensés que les plants non fauchés. Avec le cultivar local blanc ainsi que les variétés FKB-ROUGE et ICPL 87119 Asha, aucune différence significative $(\mathrm{P}>0,01)$ n'est constatée entre les plants fauchés et les plants non fauchés. Cependant, les plants fauchés ont produit moins de tanins condensés que les plants non fauchés pour chez les variétés ICPL 87119 GUIMU 3 (Asha), ICP 7035-KAMICA, ICP 8863-MARUTI et ICPL 332 ABAAYA. Au 
stade maturation, seul le cultivar local rouge et la variété FKB-ROUGE ont enregistré une teneur en tanins condensés plus élevée chez les plants fauchés comparativement aux plants non fauchés. Concernant les autres variétés et cultivars, aucune différence significative $(\mathrm{P}>0,01)$ n'est constatée entre les plants fauchés et les plants non fauchés pour les variétés ICP 8863-MARUTI, ICPL 87119 GUIMU 3 (Asha) et ICPL 332 ABAAYA. Cependant, les plants fauchés ont produit moins de tanins condensés par rapport aux plants non fauchés pour les cultivars locaux noir et blanc et les variétés ICP 7035KAMICA et ICPL 87119 Asha.

Tableau 2 : Rendements d'extraction.

\begin{tabular}{lc}
\hline Variétés/Cultivars & $\mathbf{R}(\boldsymbol{\%})$ \\
\hline Cultivar local blanc & 24,86 \\
Cultivar local rouge & 20,23 \\
Cultivar local noir & 23,6 \\
ICPL 87 119- ASHA & 23,46 \\
ICP 8863-MARUTI & 22,41 \\
ICP 7035-KAMICA & 22,18 \\
FKB-ROUGE & 22,10 \\
ICPL 87 119 GUIMU 3 (Asha) & 23,07 \\
ICPL 332 ABAAYA & 21,48 \\
\hline
\end{tabular}

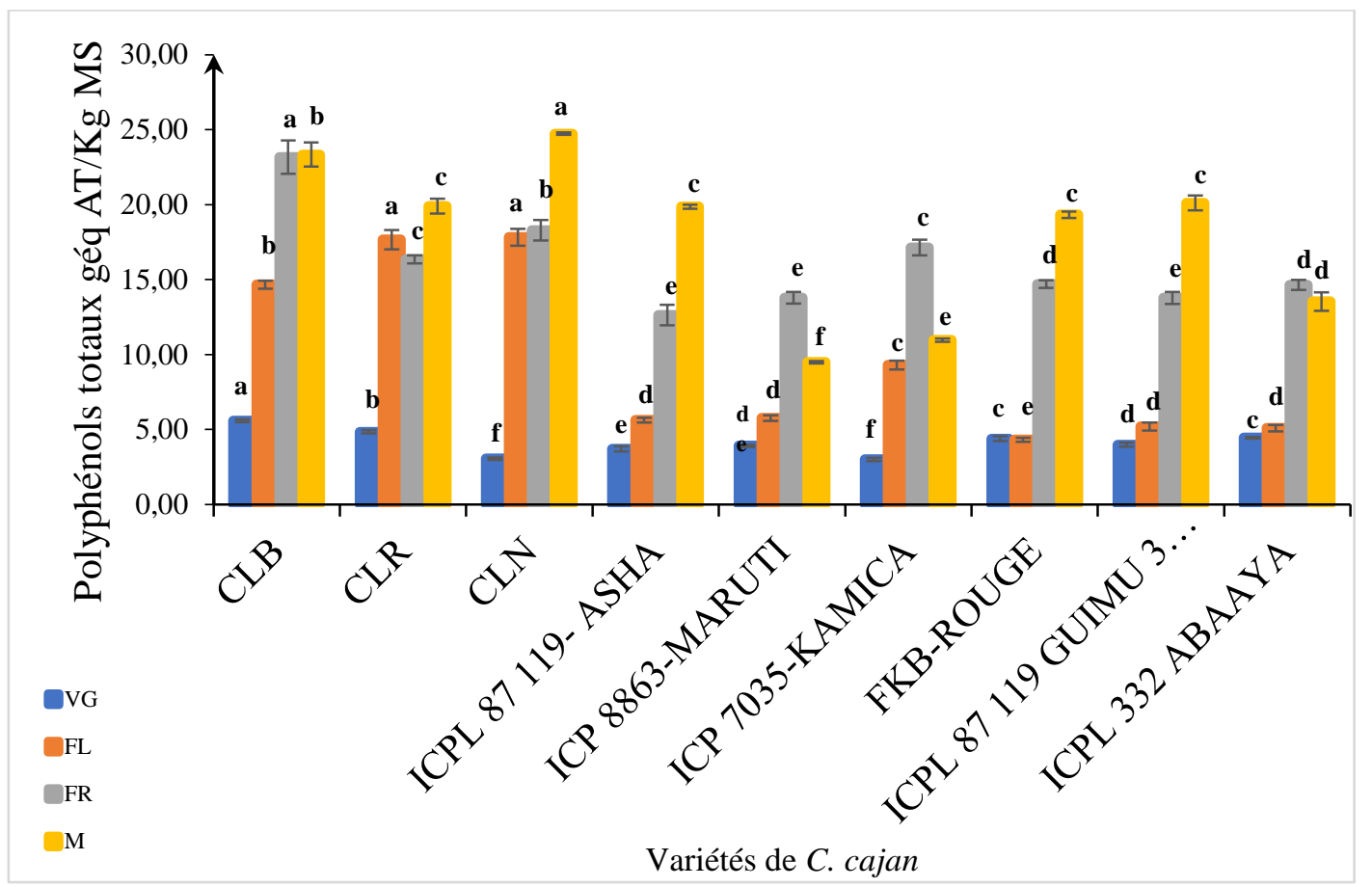

$\mathrm{AT}=$ acide tannique, $\mathrm{CLB}=$ cultivar local blanc, $\mathrm{CLR}=$ cultivar local rouge, $\mathrm{CLN}=$ cultivar local noir, $\mathrm{VG}=$ phase végétatif, $\mathrm{Fl}=$ phase floraison, $\mathrm{FR}=$ phase fructification, $\mathrm{M}=$ maturation.

Les bâtons portant les lettres différentes ( $a, b, c, d, e, f)$ pour le même stade phénologique diffèrent significativement ( $<<$ $0,01)$.

Figure 2: Polyphénols totaux des différentes variétés aux différents stades phénologiques. 


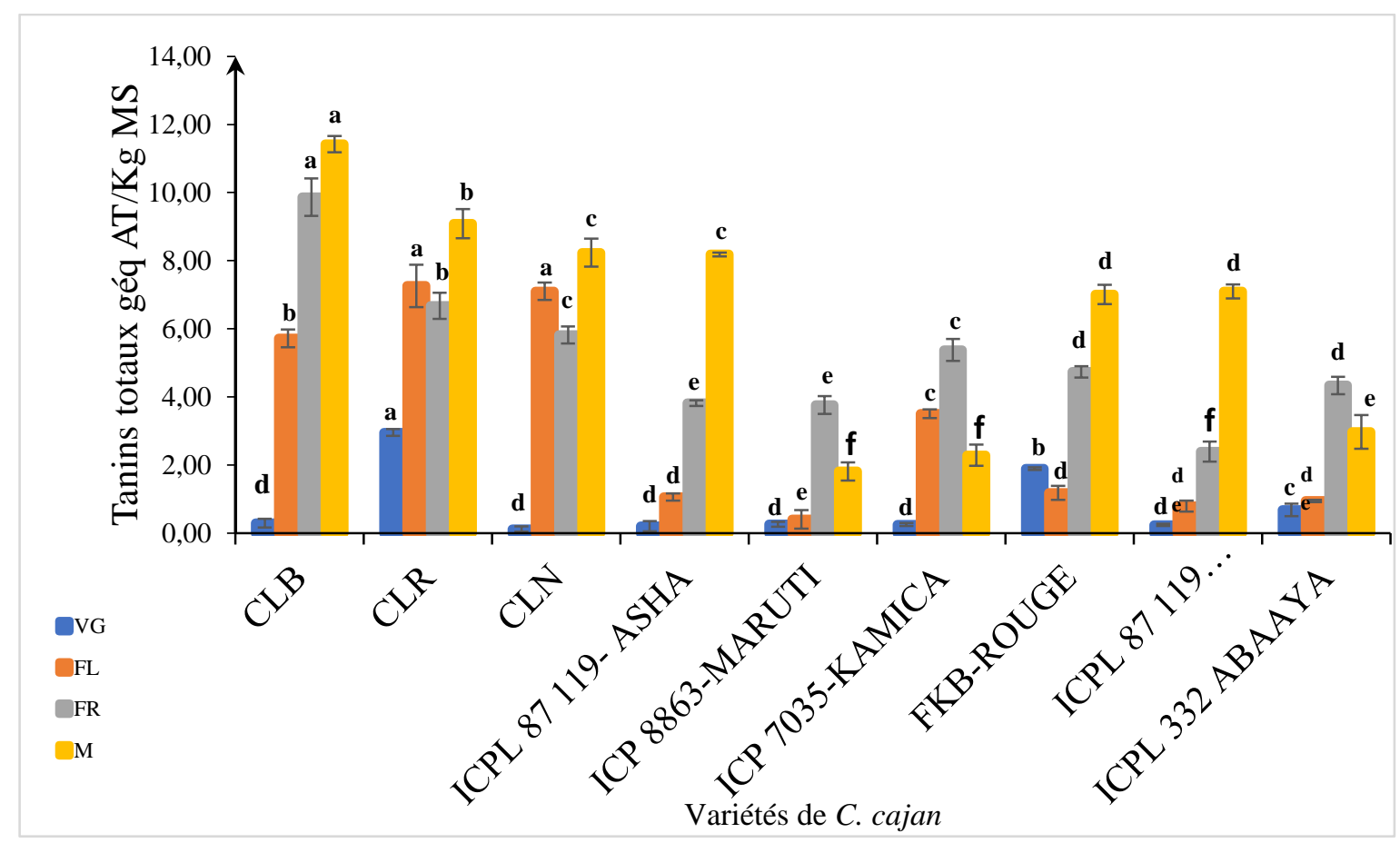

$\mathrm{AT}=$ acide tannique, $\mathrm{CLB}=$ cultivar local blanc, $\mathrm{CLR}=$ cultivar local rouge, $\mathrm{CLN}=$ cultivar local noir, $\mathrm{VG}=$ phase végétatif, $\mathrm{Fl}=$ phase floraison, $\mathrm{FR}=$ phase fructification, $\mathrm{M}=$ maturation.

Les bâtons portant les lettres différentes ( $\mathrm{a}, \mathrm{b}, \mathrm{c}, \mathrm{d}, \mathrm{e}, \mathrm{f})$ pour le même stade phénologique diffèrent significativement $(\mathrm{p}<$ 0,01 ).

Figure 3: Tanins totaux des différentes variétés aux différents stades phénologiques.

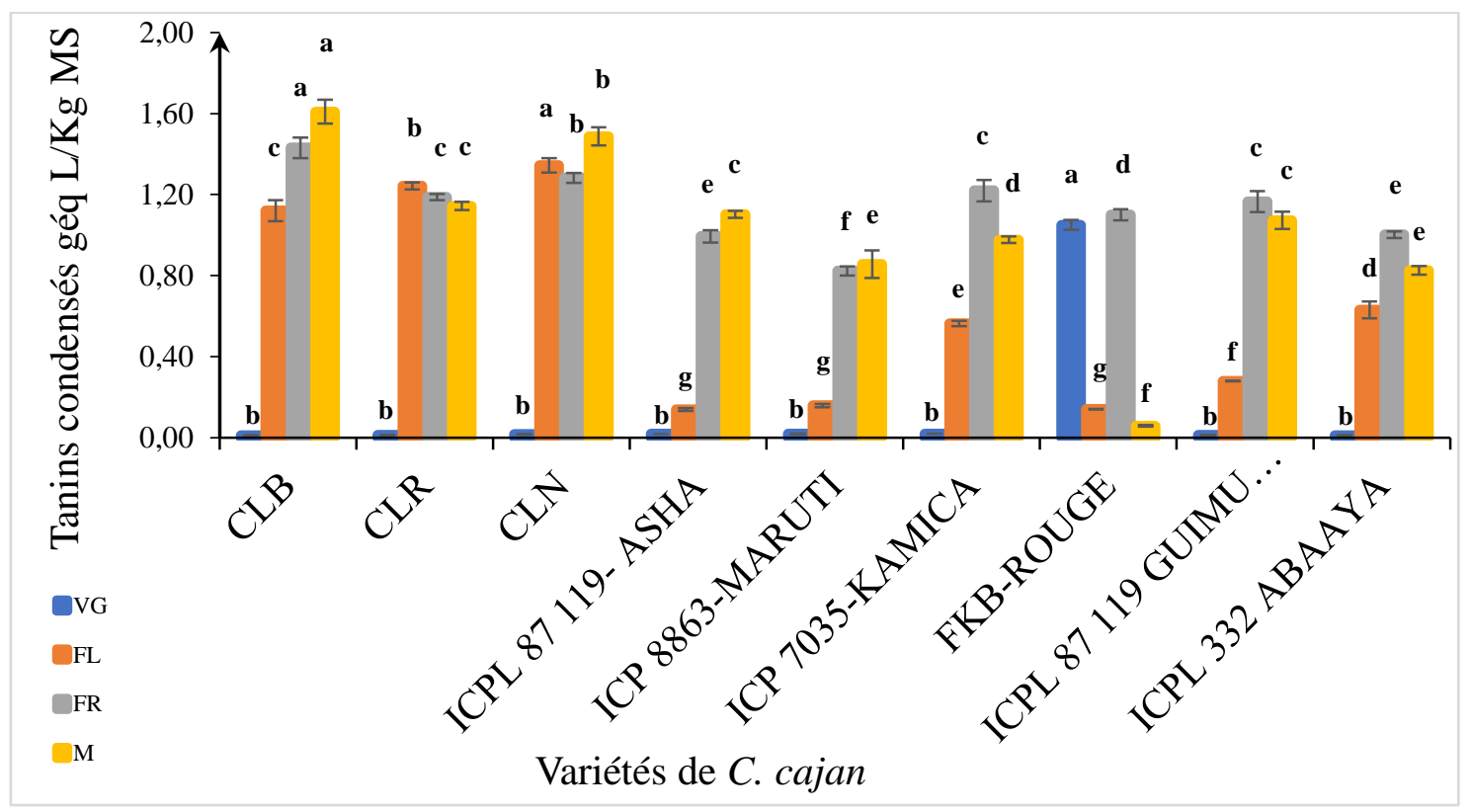

$\mathrm{L}=$ leucocyanidine, $\mathrm{CLB}=$ cultivar local blanc, $\mathrm{CLR}=$ cultivar local rouge, $\mathrm{CLN}=$ cultivar local noir, $\mathrm{VG}=$ phase végétatif, $\mathrm{Fl}=$ phase floraison, $\mathrm{FR}=$ phase fructification, $\mathrm{M}=$ maturation.

Les bâtons portant les lettres différentes ( $\mathrm{a}, \mathrm{b}, \mathrm{c}, \mathrm{d}, \mathrm{e}, \mathrm{f}, \mathrm{g})$ pour le même stade phénologique diffèrent significativement $\mathrm{p}<$ $0,05)$.

Figure 4: Tanins condensés des différentes variétés aux différents stades phénologiques. 

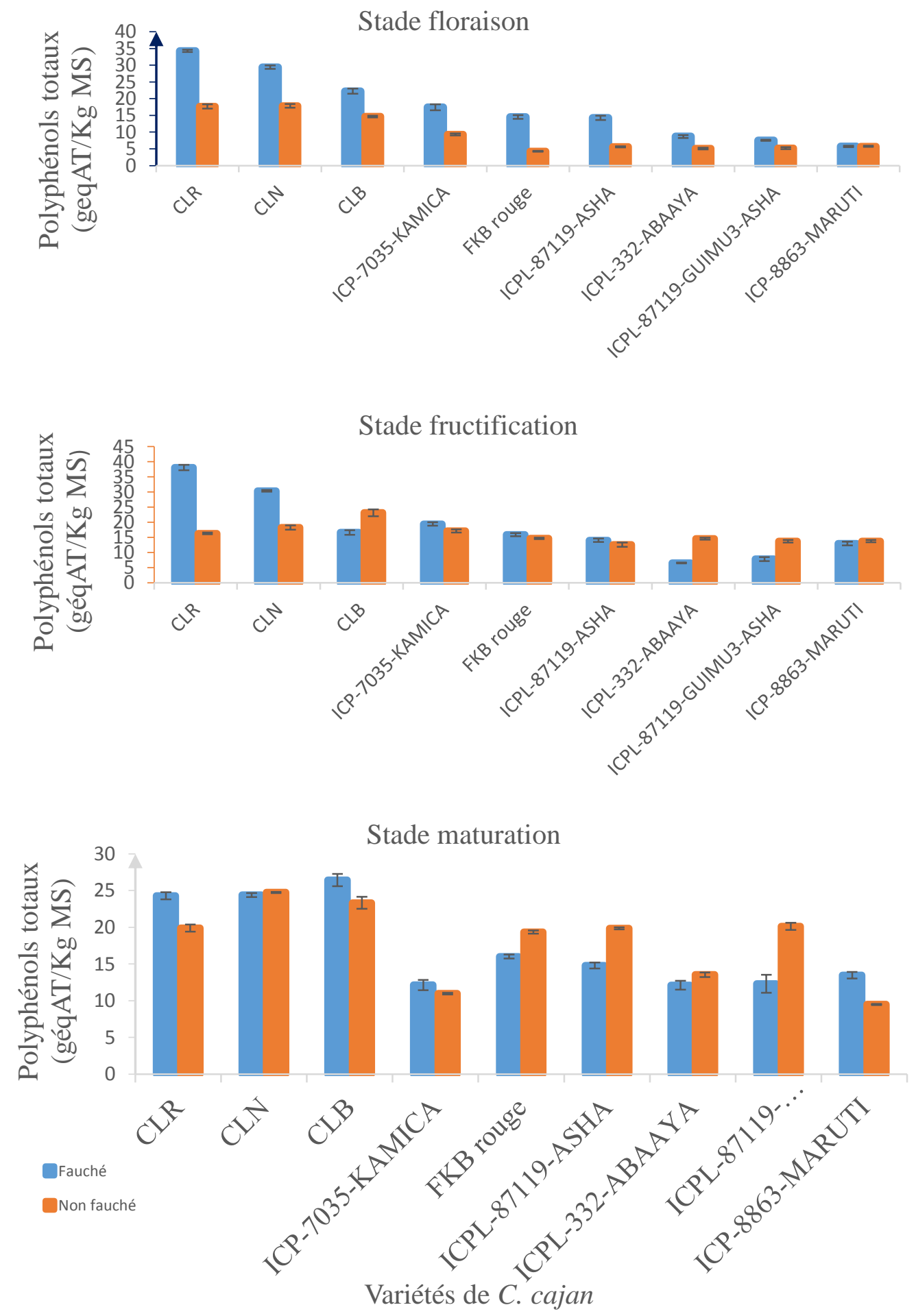

$\mathrm{AT}=$ acide tanique, $\mathrm{CLB}=$ cultivar local blanc, $\mathrm{CLR}=$ cultivar local rouge, $\mathrm{CLN}=$ cultivar local noir

Figure 5: Influence de la variété et du mode d'exploitation sur la production de polyphénols aux différents stades phénologiques. 

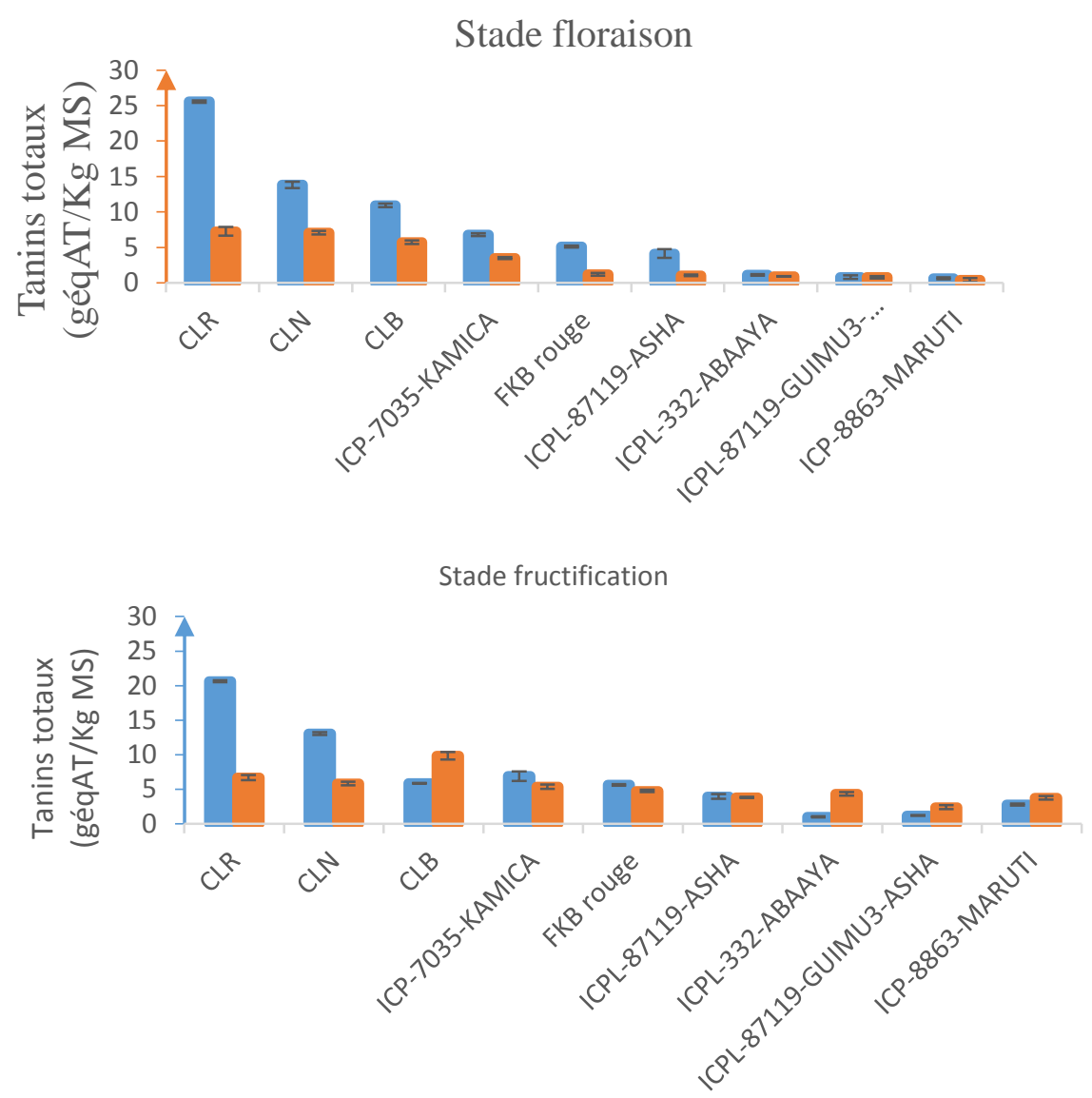

Stade maturation

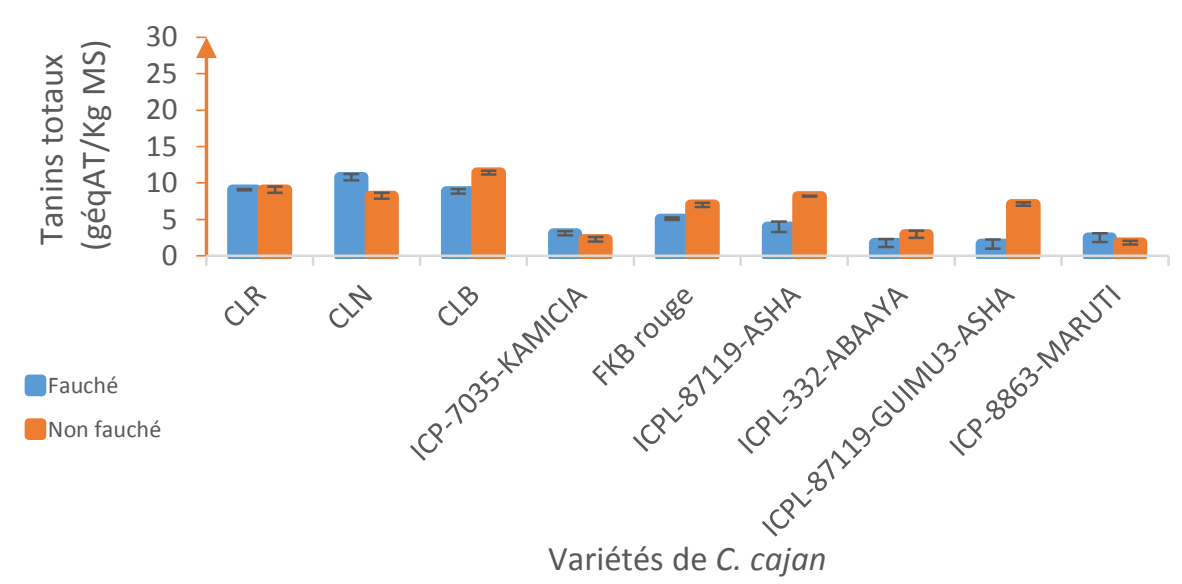

$\mathrm{AT}=$ acide tanique, $\mathrm{CLB}=$ cultivar local blanc, $\mathrm{CLR}=$ cultivar local rouge, $\mathrm{CLN}=$ cultivar local noir,

Figure 6: Influence de la variété et du mode d'exploitation sur la production de tanins totaux aux différents stades phénologiques. 

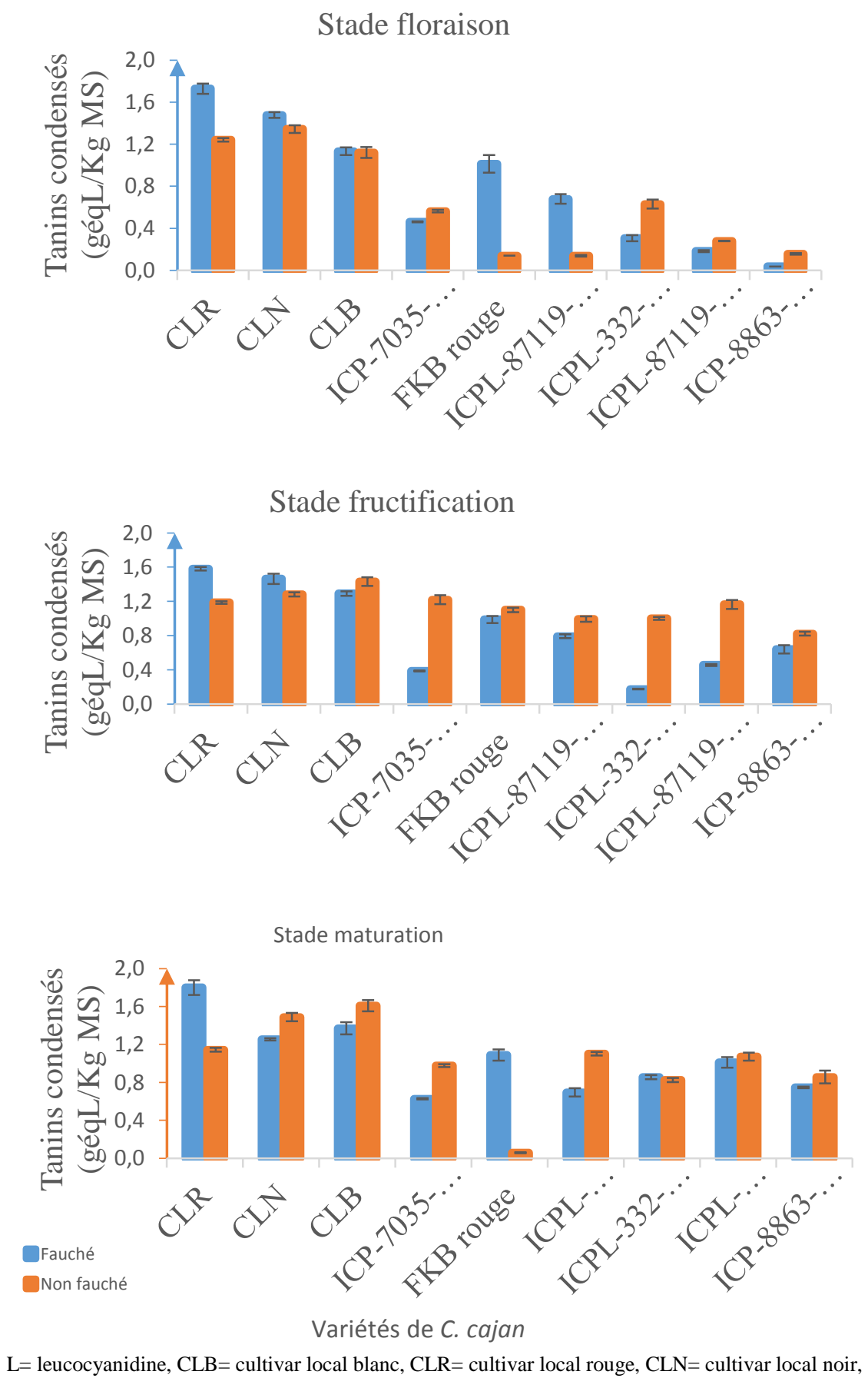

Figure 7: Influence de la variété et du mode d'exploitation sur la production de tanins condensés aux différents stades phénologiques. 


\section{DISCUSSION}

Les concentrations en polyphénols totaux des différentes variétés sont comparables à celles obtenues par différents auteurs. Fluck et al. (2013) ont obtenu $11 \mathrm{~g} / \mathrm{kg}$ MS, tandis que Vitti et al. (2005) ont trouvé $8,2 \mathrm{~g} / \mathrm{kg}$ MS. Ces concentrations demeurent tout de même inférieures à la valeur trouvée par Harris et al. (2014) qui est de $116,1 \mathrm{~g} / \mathrm{kg}$ MS.

Les teneurs en tanins totaux sont également comparables à celles de Vitti et al. (2005) et Harris et al. (2014) qui sont respectivement de $5 \mathrm{~g} / \mathrm{kg}$ MS et $4,9 \mathrm{~g} / \mathrm{kg}$ MS.

Enfin, les concentrations en tanins condensés se rapprochent des $0,5 \mathrm{~g} / \mathrm{kg}$ MS trouvés par Vitti et al. (2005). Elles demeurent néanmoins très faibles comparées au $109,6 \mathrm{~g} / \mathrm{kg}$ MS rapportés par Fondevila et al. (2002). Ces derniers auteurs ont utilisé comme standard, les tanins quebracho. Or ces tanins, lorsqu'ils sont utilisés en tant que tel, ont tendance à surestimer la teneur en tanins (Vitti et al., 2005). Ce qui pourrait expliquer la valeur élevée des tanins condensés rapportée par ces derniers. Ces valeurs en tanins condensés, bien que faibles, se rapprochent des 0,08 à $0,38 \mathrm{~g} / \mathrm{kg}$ de MS trouvées pour certains fourrages ligneux des régions semiarides du Nigéria (Njidda, 2010) et de l'intervalle de 0,41 à $0,81 \mathrm{~g} / \mathrm{kg}$ MS rapportée par Njidda et al. (2012) pour les fourrages des zones semi-arides. En référence aux valeurs seuils rapportées par Hoste et al. (2011) (40$50 \mathrm{~g} / \mathrm{kg}$ MS) et Seresinhe et Pathirana (2003) $(20-40 \mathrm{~g} / \mathrm{kg} \mathrm{MS})$, ces valeurs en tanins condensés obtenues dans le cadre de cette étude ne pourraient induire chez les petits ruminants, respectivement des effets anthelminthiques et des effets positifs sur la digestion des protéines et le gain de poids vif. Cependant, cette supposition est à prendre avec beaucoup de précautions, car les travaux de Vitti et al. (2005) et de Fluck et al. (2013) ont montré que les tanins condensés, même à de faibles concentrations, ont un impact positif sur la fermentation des légumineuses fourragères. Ces effets bénéfiques ont pour conséquences non seulement un gain de poids vif (Chala et al., 2013) mais en plus, une action indirecte sur les parasites gastrointestinaux grâce à l'amélioration de la réponse (résistance et résilience) de l'hôte contre les vers (Hoste et al., 2011). De plus, d'autres métabolites secondaires autres que les tanins, peuvent avoir des capacités de liaison aux protéines ou avoir des propriétés anthelminthiques (Hoste et al., 2006). C'est pourquoi des études ultérieures sont nécessaires pour confirmer ou non les propriétés anthelminthique, nutritionnelle et nutraceutique des différentes variétés et cultivars étudiés.

$\mathrm{Au}$ regard des différents résultats, il existe une différence variétale pour tous les paramètres mesurés. Les variétés traditionnelles ont affiché les valeurs les plus élevées aux différents stades phénologiques comparées aux variétés améliorées. Ces variétés traditionnelles sont sensibles à beaucoup de facteurs biotiques (Fusarium udum Butler, Cercospora cajani Henning, ravageurs arthropodes, etc.) et abiotiques (températures, engorgement en eau du sol, forte salinité, photopériodisme, etc.) (Sankara et al., 2012; Kaoneka et al., 2016); ce qui pourrait expliquer leurs teneurs élevées. En effet, la production de polyphénols et de tanins est une tactique de résistance contre les insectes (Sharma et al., 2009). Chez certains parents sauvages de Cajanus cajan, l'expression de la résistance à Helicoverpa armigera, insecte dévastateur des gousses a été associée à des teneurs élevées de tanins et de polyphénols (Sharma et al., 2009). Cette différence variétale a également été constatée par Azuhnwi et al. (2011) chez Onobrychis viciifolia (sainfoin). Enfin, pour chaque stade phénologique, la variété ayant présenté la teneur la plus élevée en polyphénols totaux, n'a pas forcement enregistré la valeur la plus élevée en tanins totaux et en tanins condensés.

D'une façon générale, une évolution des polyphénols totaux et des tanins au cours du cycle de développement a été constatée. Ce même constat a été fait par Li et al. (2014). Ceci tiendrait au fait que pendant la phase de croissance, les végétaux produisent beaucoup de biomasse et peu de carbone sont disponibles pour la synthèse des composés 
phénoliques et des tanins. A la floraison, qui correspond à un excès d'activité photosynthétique, il y a une faible demande en carbone pour la croissance et un excès de carbone est disponible pour la synthèse des tanins ( $\mathrm{Li}$ et al., .2014). On pourrait aussi penser au fait que les fleurs et gousses de $C$. cajan étant fortement agressées par les insectes, la plante augmente pendant la phase de reproduction, sa production en composés phénoliques et en tanins, dans le but de se protéger des attaques des insectes.

Toutefois, les cultivars locaux et les variétés ICP 7035-KAMICA, FKB-ROUGE et ICPL 87119 GUIMU 3 (Asha) ont, au cours de la phase reproductive (floraison, fructification et maturation) produit soit plus de polyphénols soit plus de tanins à la floraison qu'à la fructification et souvent même qu'à la maturation ou à la fructification qu'à la maturation. Concernant $C$. cajan, la distinction entre floraison, fructification et maturation n'est pas nette. En effet, la fructification se déroule en même temps que la floraison et même plus tard avec la maturation. Il convient donc de distinguer deux phases: la phase végétative et la phase reproductive. Ainsi on pourrait dire que les polyphénols et tanins de $C$. cajan augmentent du stade végétatif au stade reproductif. Un résultat inattendu dans le cadre de cette étude est celui de la variété FKB-ROUGE. Contrairement aux autres variétés, elle a produit plus de tanins condensés au stade végétatif qu'au stade reproductif. Ce qui laisse supposer qu'elle a subi une contrainte particulière au cours de cette phase comme par exemple une attaque d'agents pathogènes.

L'effet du mode d'exploitation (fauche ou non) sur la production de polyphénols et de tanins est un peu mitigé. Cependant, il existe pour chaque stade phénologique, une interaction entre les variétés et le mode d'exploitation. Si pour certaines variétés une augmentation des polyphénols et tanins a été observée après la fauche, pour d'autres, aucune différence n'a été observée. Pour d'autres encore, une baisse des composés phénoliques et tanins a même été observée. Ce qui est en désaccord avec nos attentes, car on s'attendait à une augmentation des composés phénoliques et des tanins pour toutes les variétés, si l'on assimile la fauche à un broutage par les herbivores. Car en effet, la production de tanins est une arme de défense des plantes contre les herbivores en modifiant le goût ou la digestibilité des aliments (Seresinhe et Pathirana, 2003; Zaidi et al., 2009).

Tous ces résultats obtenus dans cette étude suggèrent que dans les études portant sur $C$. cajan mettant en jeu les polyphénols et les tanins, la variété (ou le cultivar), le stade phénologique et le mode d'exploitation doivent être précisés; ce qui n'a pas été souvent le cas dans plusieurs travaux (Harris et al., 2014; Kaboré et al., 2016 ; Vidhya et Ahmed, 2017).

\section{Conclusion}

Le but de cette étude est d'étudier les teneurs en polyphénols totaux et en tanins des variétés de Cajanus cajan au cours de la première année de culture en rapport avec le mode d'exploitation. Il ressort au terme de cette étude qu'il existe une différence variétale chez cette espèce dans la production de ces composés secondaires. Les variétés traditionnelles présentent les concentrations les plus élevées en ces métabolites secondaires. Bien qu'ils soient faiblement produits par les différentes variétés, on observe une augmentation de leurs teneurs au cours du cycle de développement. Ces composés sont influencés à la fois par la variété, le stade phénologique et le mode d'exploitation. Ce qui indique que dans des études portant sur C. cajan et impliquant les polyphénols et des tanins, la variété (ou le cultivar), le stade phénologique et le mode d'exploitation doivent être absolument précisés. Par ailleurs, il se peut que la variabilité variétale observée dans la production de polyphénols et de tanins induise également une différence variétale dans les effets anthelminthiques, nutritionnels et nutraceutiques. Des études ultérieures devront être menées afin de confirmer ou infirmer cette hypothèse. 


\section{CONFLIT D'INTERETS}

Les auteurs déclarent qu'ils n'ont pas de conflits d'intérêts.

\section{CONTRIBUTIONS DES AUTEURS}

FPK a participé à la rédaction du protocole expérimental, à sa mise en œuvre, à la collecte des données puis à la rédaction du manuscrit. YMY a participé à la rédaction du protocole expérimental, au suivi des travaux puis à la rédaction du manuscrit. NCB a participé à la rédaction du protocole expérimental puis à la rédaction du manuscrit. KPN a réalisé les analyses chimiques puis a participé à la rédaction du manuscrit. ENW a supervisé tout le travail depuis la rédaction du projet de protocole jusqu'à la rédaction du projet de manuscrit puis à la validation du manuscrit.

\section{REMERCIEMENTS}

Les auteurs du présent manuscrit remercient le Projet de Gestion Intégré des Ranchs et Stations du Ministère des Ressources Animales et Halieutiques de Côte d'Ivoire pour avoir accepté que la Station Semencière de Yamoussoukro serve de site d'expérimentation.

\section{REFERENCES}

Azuhnwi BN, Boller B, Martens M, DohmeMeier F, Ampuero S, Gûnter S, Kreuzer M, Hess HD. 2011. Morphology, tannin concentration and forage value of 15 Swiss accessions of sainfoin (Onobrychis viciifolia Scop.) as influenced by harvest time and cultivation site. Grass Forage Sci., 66: 474-487.

DOI : https://doi.org/10.1111/j.13652494.2011.00811.x

Banfoh B. 2004. Introduction des légumineuses fourragères dans les pratqiues culturales: expérience des projets de développement du Togo. In Introduction des Plantes Fourragères dans les Systèmes de Production en Afrique de l'Ouest, Dicko M, Ehouinsou M, Aboh AB, Desquesnes M (Eds). CIRDES, PROCORDEL, actes de l'atelier tenu à Cotonou (Bénin), du 19 au 21 janvier 2004, pp 59- 65.

Bodji NC. 1997. Influence de la hauteur et du rythme de coupe sur la productivité et la pérennité d'une culture de Cajanus cajan CIAT 18700. Revue Elev. Med. Vét. Pays Trop., 50(4) : 309-312.

Chala M, Temesgen A, Tegegn G. 2013. Effect of feeding Leucaena pallida with concentrate and antihelmentic treatment on growth performance and nematode parasite infestation of Horro ewe lambs in Ethiopia. International Journal of Livestock Production, 4(10): 155-160. DOI: $10.5897 / \mathrm{IJLP} 2013.0176$

Fluck AC, Kozloski GV, Martins AA, Mezzomo MP, Zanferari F, Stefanello S. 2013. Relationship between chemical components, bacterial adherence and in vitro fermentation of tropical forage legumes. Ciênc. agrotec., Lavras, 37 (5) : 457-464. DOI: http://dx.doi.org/10.1590/S141370542013000500010.

Fondevila M, Nogueira-Filho JCM, BarriosUrdanata A. 2002. In vitro microbial fermentation and protein utilisation of tropical forage legume grown during the dry season. Anim. Feed and Technology, 95: 1-14. DOI: https://doi.org/10.1016/S03778401(01)00315-7

Girard M, Grosse BA, Ampuero KS, Arrigo Y, Wyss U, Kreuzer M, Dohme-Meier F, Bee G. 2013. Le mode de conservation $\mathrm{du}$ fourrage et le cycle influent sur la teneur en tanins condensés des légumineuses. Renc. Rech. Ruminants, 20: 99.

Harris KK, Sahu M, Verma D. 2014. Phytochemical analysis of the leaf, stem and seed extracts of Cajanus cajan L. (Dicotyledoneae: Fabaceae). World Journal of Pharmacy and Pharmaceutical Sciences, 3 (8): 694733.

Hoste H, Jackson F, Athanasiadou S, Thamsborg SM, Hoskin SO. 2006. The effects of tannin-rich plants on parasitic nematodes in ruminants. TRENDS in 
Parasitology, 22(6): 253-261. DOI: 10.1016/j.pt.2006.04.004

Hoste H, Manolaraki F, Brunet S, Arroyo López C, Martínez-Ortiz de Montellano C, Sotiraki S, Torres Acosta F. 2011.The anthelmintic properties of tannin-rich legume forages: from knowledge to exploitation in farm conditions. In Challenging Strategies to Promote the Sheep and Goat Sector in the Current Global Context, Ranilla MJ, Carro MD, Ben Salem H, Moran d-Fehr P (eds.). Zaragoza: CIHEAM / CSIC / Universidad de León / FAO ; 295-304 (Option s Méditerranéennes: Série A. Séminaires Méditerranéens; $n^{\circ}$. 99).

Hoste H, Torres-Acosta JFJ, Sandoval-Castro CA, Mueller-Harvey I, Sotiraki S, Louvandini $\mathrm{H}$, Thamsborg SM, Terrill TH. 2015. Tannin containing legumes as a model for nutraceuticals against digestive parasites in livestock. Veterinary Parasitology, 212: 5-17. DOI:

http://dx.doi.org/10.1016/j.vetpar.2015.0 6.026

Kabore A, Konate A, Gnanda BI, Yougbare B, Traore A, Teguera A, Diarra S, Tamboura HH, Belem AMG. 2016. Seed germination and anthelmintic activity of Cajanus cajan on sheep. Journal of Chemical and Pharmaceutical Research, 8 (1): 403-410.

Kaoneka RS, Saxena KR, Silim NS, Odeny AD, Rao GRPVN, Shimelis AH, Siambi M, Varshney KR. 2016. Pigeonpea breeding in eastern and southern Africa: challenges and opportunities. Plant Breeding, 135: 148-154. DOI : 10.1111/pbr.12340

Koutouan FP, Bodji NC, Wandan EN, Ta Bi D B. 2017. Effet de la fertilisation phospho-potassique sur le rendement grainier et la qualité des semences de Cajanus cajan L. Millsp. sur un ferrasolà Yamoussoukro, région centre de la Côte d'Ivoire. European Scientific Journal, 21(13): $\quad$ 1857-7881. DOI: 10.19044/esj.2017.v13n21p7
Li Y, Iwaasa AD, Wang Y, Jin L, Han G, Zhao M. 2014. Condensed tannins concentration of selected prairie legume forages as affected by phenological stages during two consecutive growth seasons in western Canada. Can. J. Plant Sci. 94: 817-826. DOI: 10.4141/CJPS2013-234.

Makkar HPS. 2000. Quantification of tannins in tree foliage. A laboratory manual for the FAO/IAEA co-ordinated research project on 'Use of Nuclear and Related Techniques to Develop Simple Tannin Assays for Predicting and Improving the Safety and Efficiency of Feeding Ruminants on Tanniniferous Tree foliage. FAO/IAEA working document IAEA, VIENA (Austria), $14 \mathrm{p}$.

N'draman-donou E, Fofié Y, Adjambri E, Mélèdje MF, Sawadogo D. 2015. Caractérisation et évaluation in vitro de l'effet antifalcimiant des graines de Cajanus cajan (Fabacées) sur les drépanocytes à Abidjan - Côte d'Ivoire. Int. J. Biol. Chem. Sci., 9 (5): 2300-2308. DOI: http://dx.doi.org/10.4314/ijbcs.v9i5.4

Njidda AA. 2010. Determining Dry Matter Degradability of Some Semi-Arid Browse Species of North-Eastern Nigeria Using the In Vitro Technique. Nigerian Journal of Basic and Applied Science, 18(2): 160-167. DOI: http://dx.doi.org/10.4314/njbas.v18i2.64 306

Njidda AA, Olatunji EA, Raji AY. 2012. Semi arid browse forages: Their antinutritive substances and in sacconeutral detergent fibre and organic matter degradability. Journal of Agriculture and Veterinary Science, 1(6): 21-30.

Ogni CA, Kpodekon MT, Dassou HG, Boko C K, Koutinhouin BG, Dougnon JT, Youssao AKI, Yedomonhan $\mathrm{H}$, Akoegninou A. 2014. Inventaire ethnopharmacologique des plantes utilisées dans le traitement des pathologies parasitaires dans les élevages extensifs et semi-intensifs du Bénin. Int. J. Biol. 
Chem. Sci., 8(3): 1089-1102. DOI: http://dx.doi.org/10.4314/ijbcs.v8i3.22

Onana J, Ze MA, Sadou I, Awa AA, Mainam F, Guibert H, Mvondo AJ-P, Tarla NF. 2007. Impact des légumineuses fourragères et/ou de couverture sur la biodiversité floristique au NordCameroun. Int. J. Biol. Chem. Sci., 1(2): 165-175.

DOI: http://dx.doi.org/10.4314/ijbcs.v1i2.3968 7

Pal D, Mishra P, Sachan N, Ghosh AK. 2011. Biological activities and medicinal properties of Cajanus cajan (L) Millsp. $J$ Adv Pharm Technol Res., 2(4): 207-214. DOI: $10.4103 / 2231-4040.90874$

Sankara F, Dabiré BCL, Dugravot S, Cortesero MA, Sanon A. 2012. Capacités de discrimination des femelles de quatre souches de Callosobruchus maculatus Fab. (Coléoptera : Chrysomelidae, Bruchinae) pour la localisation de l'hôte et la ponte aux dépens d'hôtes secondaires. Int. J. Biol. Chem. Sci., 6(3): 1303-1315. DOI: http://dx.doi.org/10.4314/ijbcs. v6i3.33

Seresinhe T, Pathirana KK. 2003. Forage tannins in ruminant nutrition. Tropical Agricultural Research and Extension, 6: 29-43.

Sharma HC, Sujana G, Rao MD. 2009. Morphological and chemical components of resistance to pod borer, Helicoverpa armigera in wild relatives of pigeonpea. Arthropod-Plant Interactions, 3: 151161. DOI: 10.1007/s11829-009-9068-5
Shenkute B, Hassen A, Ebro A, Amen N. 2013. Performance of Arsi-Bale kids supplemented with graded levels of pigeonpea in dry season in Mid Rift valley of Ethiopia. Afr. J. Agric. Res., 8(20): $\quad 2366-2370 . \quad$ DOI: 10.5897/AJAR11.1533

Vidhya AT, Ahmed JS. 2017. Preliminary Phytochemical and Trace metal Screening from Cajanus cajan (L.) Millsp. Asian Journal of Pharmaceutical Technology \& Innovation, 05(23): 01-06. www.asianpharmtech.com

Vitti dMSS, Abdalla AL, Bueno ICS, Silva Filho JC, Costa C, Bueno MS, Nozella EF, Longo C, Vieira EQ, Cabral Filho SLS, Godoy PB, Mueller-Harvey I. 2005. Do all tannins have similar nutritional effects? A comparaison of three brazilian fodder legumes. Anim. Feed Sci. Technol., 119(3-4): 345-361. DOI: 10.1016/j.anifeedsci.2004.06.004

Yadav K, Yadav KS, Yadav A, Pandey PV, Dwivedi. NU. 2012. Genetic Diversity of Pigeonpea (Cajanus cajan (L.) Millsp.) Cultivars and Its Wild Relatives Using Randomly Amplified Polymorphic DNA (RAPD) Markers. American Journal of Plant Sciences, 3: 322-330. DOI: http://dx.doi.org/10.4236/ajps.2012.3303 8

Zaidi F, Hassissene N, Allouache H, Kichou M, Ourdani S, Rezki K, Bellal MM, Grongnet JF, Youyou A. 2009. Les composés phénoliques, facteur limitant $\mathrm{du}$ grignon d'olive chez les ruminants. Revue Méd. Vét., 160(2) : 67-73. 